\title{
Discussão da aplicação do conceito de City Information Modelling (CIM) em cidades consolidadas'
}

\section{Discussion of the application of the concept of City Information Modelling (CIM) in consolidated cities}

\author{
Souza, Letícia; Bueno, Cristiane² \\ 1 Universidade Federal de São Carlos - UFSCar, Rodovia Washington Luís (SP- \\ 301), km 235, CEP 13565-905, São Carlos - SP, Brasil e-mail: \\ leticia.souza1707@gmail.com \\ 2 Universidade Federal de São Carlos - UFSCar, e-mail: cbueno@ufscar.br
}

\begin{abstract}
RESUMO
O conceito de City Information Modelling (CIM), definido por Thompson et al. (2016) como a aplicação prática dos dados digitais em relação a gestão e planejamento da cidade colaborando com os cidadãos, foi apresentado inicialmente por Khemlani (2005) onde abordava o tema como uma extensão do conceito Building Information Modelling (BIM) para cidades, onde auxiliasse de forma mais eficaz a lidar com desastres naturais, contribuindo para uma réplica digital e precisa da cidade, que pudesse ser submetida a simulações de impactos de decisões e análises holísticas. Em paralelo, o conceito de Cidades Inteligentes que, segundo a União Europeia, é um lugar onde as redes e serviços tradicionais se tornam mais eficientes com o uso de Tecnologias de Informação e Comunicação (TIC) em benefício de sua população, surgiu em decorrência dos grandes desafios sociais como o aumento da população urbana, necessidades de transporte, saúde e segurança (ABELLA et al., 2017). O presente trabalho pretende explorar as potenciais aplicações do conceito CIM em cidades de urbanização consolidada, relacionado ao conceito de Cidades Inteligentes. Busca-se contribuir para o entendimento das potencialidades de implementação da plataforma em cidades de urbanização consolidada visando soluções tecnológicas e sustentáveis que atendam às necessidades locais.
\end{abstract}

Palavras-chave: City Information Modelling, Cidades Inteligentes, Ferramentas de Gestão Urbana.

\begin{abstract}
The City Information Modelling (CIM) defined by Thompson et al. (2016) as the practical application of the digital data in relation to the city planning and management collaborating with the citizens, initially presented by Khemlani (2005), where he approached the theme as an extension of the Building Information Modelling (BIM) more effectively to deal with natural disasters, contributing to a precise and digital replica of the city, which could be subjected to

\footnotetext{
${ }^{1}$ Souza, Letícia; Bueno, Cristiane. Discussão da aplicação do conceito de City Information Modelling (CIM) em cidades consolidadas. In: II SIMPÓSIO NACIONAL DE GESTÃO E ENGENHARIA URBANA: SINGEURB, 2019, São Paulo. Anais... Porto Alegre: ANTAC, 2019.
} 
simulations of holistic decision making and analysis. In parallel, the Intelligent Cities concept, which according to the European Union is a place where traditional networks and services become more efficient with the use of Information and Communication Technologies (ICT) for the benefit of its population, which arose as a result of the great challenges such as the increase of the urban population, transportation needs, health and safety (ABELLA et al., 2017). The present work intends to explore the potential applications of the CIM concept in cities of consolidated urbanization related to the concept of Intelligent Cities. It seeks to contribute to the understanding of the potential of implementing the platform in cities of consolidated urbanization aiming at technological and sustainable solutions that meet the local needs.

Keywords: City Information Modelling, Smart Cities, Urban Management Tools.

\section{INTRODUÇÃO}

O conceito de Cidades Inteligentes surgiu como uma reação aos problemas que desqualificam a vida no espaço urbano. A ONU através do Grupo Focal da União Internacional de Telecomunicações (UIT) definiu que Cidades Inteligentes e Sustentáveis são cidades inovadoras que utilizam Tecnologias de Informação e Comunicação (TIC) para melhorar a qualidade de vida, para o atendimento das gerações atuais levando em consideração os aspectos ambientais, sociais, culturais e econômicos (ONION, 2015).

Um estudo realizado por Komninos et al. (2015) revela que o potencial da maioria de aplicativos de cidades inteligentes é relativamente pequeno e demonstra pequenas mudanças nas cidades, pois tratam de problemas específicos e pouco abrangentes, sem grandes impactos.

Sendo assim, nota-se a necessidade da criação de sistemas que abranjam a cidade como um todo, facilitando assim uma visão holística. A implementação que vem sendo discutida é o uso do City Information Modelling (CIM) que, de acordo com Xu et al. (2014), deve ser um sistema eficiente de gerenciamento, que integre seus dados de maneira sistêmica e multifuncional, e permita alcançar um compartilhamento em tempo real com foco na colaboração interdisciplinar. Amorim (2016a) revela que o CIM pode ser uma alternativa para gestões urbanas, e traz à tona a sua viabilidade para resoluções de questões como a da mobilidade urbana, a crise da água, de energia, dentre outras, fazendo com que esta discussão ganhe uma posição de destaque e não podendo ser postergada.

O trabalho proposto pretende abordar as inter-relações entre os conceitos de Cidades Inteligentes e CIM para, posteriormente, contribuir para o entendimento das potencialidades de implementação da plataforma em cidades de urbanização consolidada visando soluções tecnológicas e sustentáveis que atendam às necessidades locais.

\section{OBJETIVOS E METODOLOGIA}

O objetivo central do presente trabalho é identificar as possibilidades de contribuição da plataforma CIM combinada com o uso de tecnologias inteligentes em cidades com urbanização consolidada, com foco na implementação de iniciativas que as tornem mais inteligentes.

A abordagem da pesquisa será qualitativa tendo em vista as definições de Miguel et al. (2012) uma vez que buscará, através da análise de trabalhos relevantes na área, interpretar e descrever experiências na implementação do CIM, tecnologias de informação para o planejamento urbano ou iniciativas relacionadas ao conceito de Cidades Inteligente em cidades consolidadas. A pesquisa realizada neste trabalho pode ser definida segundo Gil (2010) como exploratória tendo em vista o levantamento do estado da arte sobre o tema com o objetivo de proporcionar maior familiaridade e construir hipóteses, realizando coletas de dados de levantamentos bibliográficos e análise de exemplos para estimular a compreensão. 


\section{POTENCIALIDADES DA IMPLEMENTAÇÃO DA PLATAFORMA CIM EM CIDADES CONSOLIDADAS}

O CIM foi abordado inicialmente por Khemlani (2005) como uma extensão do conceito Building Information Modelling (BIM) mas para cidades, que auxiliasse em uma forma mais eficaz de lidar com desastres naturais, contribuindo para que tenhamos uma réplica digital, precisa e detalhada da cidade, que pudesse ser submetida a simulações de impactos de decisões e análises mais holísticas.

O autor Amorim (2015) apresenta a representação de uma "cidade virtual" baseada no conceito de Cidade Inteligente, onde a plataforma CIM - aberta aos usuários da população e administração da cidade - atua como o suporte, operado por planejadores, projetistas e administradores, como base para a sustentação da gestão urbana. Nesse modelo de cidade, as TICs ficam incumbidas de uma grande responsabilidade, pois podem contribuir para a provisão e gerenciamento de informações para o monitoramento e gestão dos serviços e infraestrutura, contribuindo também para o estreitamento dos laços entre a população e o poder público.

Ainda segundo Amorim (2016b) para que se atinja o status de Cidade Inteligente, é necessário que todos os serviços urbanos tenham a mais alta qualidade, pois a simples utilização das TICs não pode transformar a cidade. Tal fato também é abordado por Komninos et al. (2015), pois sem a infraestrutura necessária, essas aplicações têm um alcance muito limitado devido à sua atuação pontual, evidenciando a precariedade da prestação de serviços.

\subsection{Iniciativas de aplicação da plataforma CIM}

Existem algumas iniciativas de uso da plataforma CIM. No Quadro 1 serão exemplificados alguns casos em que a plataforma auxilia em diferentes subsistemas da cidade. 
Quadro 1 - Estudos de casos abordados

\begin{tabular}{|c|c|}
\hline Estudos de casos & Objetivos de aplicação \\
\hline $\begin{array}{c}\text { Estudo de caso Al } \\
\text { Identificação de } \\
\text { tubulações subterrâneas }\end{array}$ & $\begin{array}{c}\text { Trata-se de um mapeamento de tubulações subterrâneas } \\
\text { existentes na cidade de Chicago }\end{array}$ \\
\hline $\begin{array}{c}\text { Estudo de caso B) } \\
\text { Estimativa de demanda } \\
\text { de energia térmica }\end{array}$ & $\begin{array}{l}\text { Trata-se de um estudo de demanda de energia térmica para } \\
\text { eliminação de gastos de energia para aquecimento de água } \\
\text { para um conjunto de edificios residenciais }\end{array}$ \\
\hline $\begin{array}{c}\text { Estudo de caso C) } \\
\text { Melhoria de tomada de } \\
\text { decisăo em emergências } \\
\text { de incêndio }\end{array}$ & $\begin{array}{l}\text { Demonstra a utilização da plataforma CIM para o caso de uma } \\
\text { emergência de incêndio no campus da Universidade de } \\
\text { Ciência e Tecnologia de Huazhong na cidade de Hefei na China }\end{array}$ \\
\hline $\begin{array}{l}\text { Estudo de caso D) } \\
\text { Comunicação de } \\
\text { melhorias na rota do } \\
\text { tráfego }\end{array}$ & $\begin{array}{c}\text { Utilização da plataforma para demonstração a população as } \\
\text { melhorias nas rotas de trafego feitas na cidade por meio da } \\
\text { plataforma CIM }\end{array}$ \\
\hline $\begin{array}{l}\text { Estudo de caso E) } \\
\text { Simulação de } \\
\text { crescimento urbano }\end{array}$ & $\begin{array}{l}\text { Estudo de implantação de rede de transporte público e } \\
\text { estações estrategicamente alocadas em locais adensados }\end{array}$ \\
\hline $\begin{array}{c}\text { Estudo de caso Fl } \\
\text { Cálculo da energia solar } \\
\text { recebida }\end{array}$ & $\begin{array}{l}\text { Estimativa de energia recebida por meio de luz solar para } \\
\text { melhoria do isolamento térmico e de demanda de resfriamento } \\
\text { mecânico }\end{array}$ \\
\hline
\end{tabular}

Fonte: Os autores (2019)

No estudo de caso "A)" um dos mais proeminentes da implantação da plataforma CIM é o da cidade de Chicago, que está sendo modelada utilizando as plataformas Cityzenith e Reconstruct. Segundo Sherr (2017), tal iniciativa se deveu, inicialmente, aos problemas associados a redes de tubulações subterrâneas, que têm sido um problema para construtores, devido aos registros incompletos e desatualizados. Para solucionar o problema, a cidade, através de uma parceria público-privada chamada UI Labs ("Universidade + Indústria"), tem realizado um mapeamento tridimensional, através da localização de tubulações e filmagem no nível da rua, posteriormente inseridas na plataforma Reconstruct, utilizando a tecnologia de nuvens de pontos para obter o equivalente a um escaneamento do subsolo de vários ângulos, que permite converter essas informações em um modelo digital codificado por cores, determinando rotas, conexões e extensão de tais redes.

O autor Schiefelbein (2015), no estudo de caso "B)", traz o uso da plataforma CIM focado em edifícios e sistemas de energia, com o objetivo de estimar a demanda de energia térmica de um bairro residencial após retrofit. Para a análise foi utilizado os software para a retirada e cruzamento de dados seguindo a metodologia mostrada na Figura 1. 
Figura 1 - Fluxo de trabalho de parametrização do edifício do estudo de caso B)

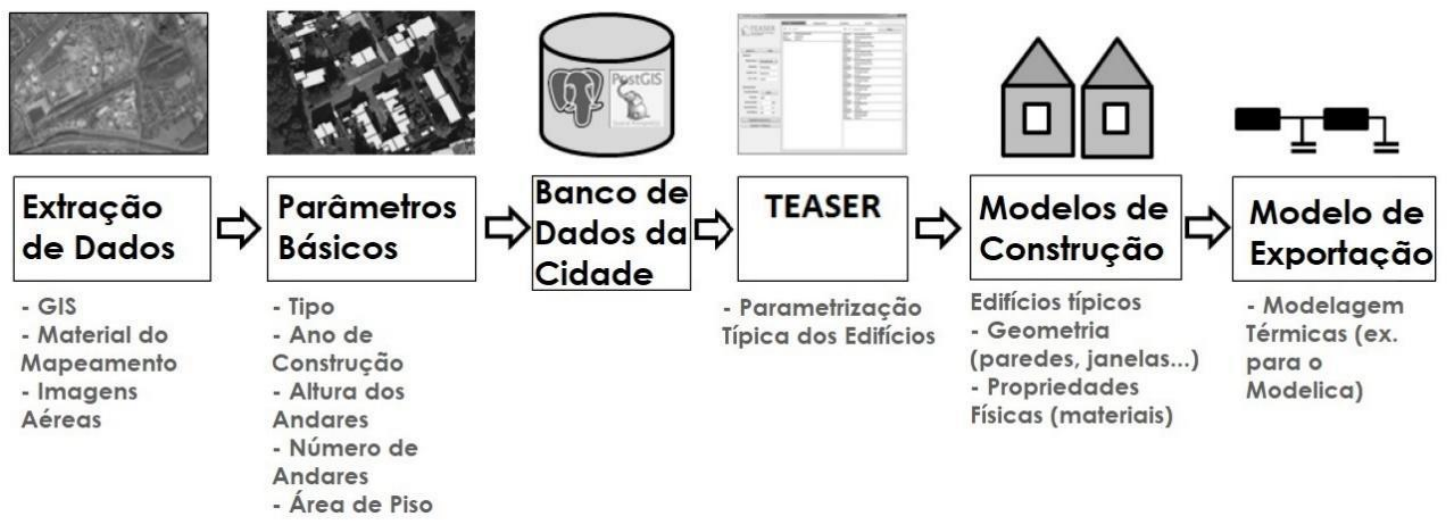

Fonte: Adaptado Schiefelbein (2015)

O caso foi realizado em um distrito residencial existente com 248 edifícios, que foram construídos antes de 1950. Como resultado do estudo após o retrofit, a demanda de energia térmica final seria ajustada pelo clima, a quantidade de energia gasta para água quente doméstica seria eliminada, e a eficiência da caldeira considerada, para gerar um valor de referência para a demanda térmica líquida de aquecimento (SCHIEFELBEIN, 2015).

O estudo de caso "C)" foi tratado no artigo de Xu et e al. (2014) onde a plataforma CIM é utilizada como exemplo na emergência de incêndio no campus da Universidade de Ciência e Tecnologia de Huazhong em Hefei, capital da província de Anhui, na China, e mostra com base na prevenção de incêndios da gestão de segurança de campus, como reduzir a ameaça do fogo, e como realizar um plano de resgate. Neste exemplo, o autor divide o caso de emergência de incêndio em quatro fases: fase de alarme, fase de decisão, fase de ação e fase de reconstrução. Em cada fase, os operadores precisam de diferentes informações que devem ser atualizadas e mantidas continuamente.

A plataforma CIM imediatamente transmite via rádio as informações de fuga aos usuários que estão dentro da escola, e emite a notificação de emergência ao posto de bombeiros mais próximo. O sistema CIM associado ao aplicativo desenvolvido, que permite calcular a melhor e mais rápida rota de resgate, através de tráfego em tempo real da cidade e verificar as condições da estrada, transmite a informação ao posto de bombeiros que irá realizar o resgate. O corpo de bombeiros de Hongshan pode avaliar a gravidade deste caso de incêndio através das câmeras e imagens em tempo real, comparando com outros casos ocorridos, para então verificar a quantidade necessária de equipes e veículos de resgate. No momento em que a equipe de resgate entra em campo, o bombeiro já teria a localização do hidrante mais próximo. Na reconstrução após o desastre, os construtores poderiam extrair as informações de construção do sistema CIM, o que poderia economizar tempo e custo de projeto (XU et al., 2014).

Reitz e Schubiger-Banz (2014) apresentam três cenários de utilização de CIM:

Uma cidade alemã de médio porte, tratada no estudo de caso "D)", que tem tido dificuldades em comunicar as recentes melhorias na rota do tráfego no centro da cidade, apresenta como solução um aplicativo em uma grande mesa multi-touch para audiências públicas, mostrando como essa ferramenta permite que não-especialistas explorem interativamente opções como, adicionar uma faixa ou alterar seu sentido de direção. Possibilita visualizar diretamente as consequências, como engarrafamentos, taxas de acidentes, e assim pode-se analisar situações em um único cruzamento ou impactos em grandes áreas.

Uma Simulação de crescimento urbano, referente ao estudo de caso "E)", em uma cidade asiática que espera um aumento na população de 500.000 pessoas até 2050, devido ao crescimento econômico da região. Um cenário é baseado em um sistema de transporte público, com zonas adensadas e próximas às estações de transporte. Projeta-se no CIM uma 
rede ao longo do litoral, para que a plataforma ajude a minimizar os custos dessa construção e otimizar as rotas, de forma que, após uma análise de caminhabilidade a plataforma fornecerá dicas para a localização das estações. Também são simuladas diferentes regras de zoneamento, para que haja um equilíbrio de residências multifamiliar, unifamiliar e espaços verdes. A partir dessa simulação são selecionados alguns projetos e apresentados para votação.

O estudo de caso "F)" aborda a realização de cálculo da energia que um edifício receberá do entorno por meio da incidência solar direta e calor refletido, para otimizar a proteção, o isolamento, o uso de energia renovável, e a necessidade de resfriamento. Utilizando dados locais abertos, é possível exportar um modelo de seu novo edifício, posicioná-lo corretamente em sua localização e a visualização tridimensional do entorno.

O Quadro 2 demonstra as potencialidades e limitações de aplicação do CIM em cidades consolidadas, baseado nos estudos de casos apresentados.

Quadro 2 - Potencialidades e limitações identificadas nos estudos de casos

\begin{tabular}{|c|c|c|}
\hline Aplicação & Potencialidades & Limite de aplicaçăo \\
\hline $\begin{array}{c}\text { Estudo de caso A| } \\
\text { Identificação de } \\
\text { tubulações subterrâneas }\end{array}$ & $\begin{array}{c}\text { Monitoramento de redes, verificação de } \\
\text { manutenção e apoio a tomada de } \\
\text { decisão de novas redes }\end{array}$ & $\begin{array}{l}\text { Realizar o levantamento } \\
\text { das tubulações existentes. }\end{array}$ \\
\hline $\begin{array}{c}\text { Estudo de caso Bl } \\
\text { Estimativa de demanda } \\
\text { de energia térmica }\end{array}$ & $\begin{array}{c}\text { Apoio a tomada de decisão de melhores } \\
\text { sistemas e monitoramento de uso }\end{array}$ & $\begin{array}{c}\text { Estimar a quantidade de uso } \\
\text { de água quente da } \\
\text { população local }\end{array}$ \\
\hline $\begin{array}{c}\text { Estudo de caso C) } \\
\text { Melhoria de tomada de } \\
\text { decisăo em } \\
\text { emergências de } \\
\text { incêndio }\end{array}$ & $\begin{array}{l}\text { Mitigar possiveis danos e perigos a } \\
\text { comunidade com um resgate mais } \\
\text { rápido e preciso. }\end{array}$ & $\begin{array}{c}\text { Realizar a parceria com } \\
\text { órgăos de diferentes esferas } \\
\text { para instalaçăo do sistema } \\
\text { com centralização de } \\
\text { dados }\end{array}$ \\
\hline $\begin{array}{c}\text { Estudo de caso D) } \\
\text { Comunicação de } \\
\text { melhorias na rota do } \\
\text { tráfego }\end{array}$ & $\begin{array}{c}\text { Auxilio na elaboração de Estudo de } \\
\text { Impacto de Vizinhança (EIV) e de Polos } \\
\text { Geradores de Tráfego (PGT) e auxilio na } \\
\text { tomada de decisão de mudanças no } \\
\text { trânsito }\end{array}$ & $\begin{array}{l}\text { Realizar o levantamento de } \\
\text { dados reais do local }\end{array}$ \\
\hline
\end{tabular}

Fonte: Os autores (2019)

Quadro 2 - Potencialidades e limitações identificadas nos estudos de casos (Continuação)

\begin{tabular}{|c|c|c|}
\hline $\begin{array}{c}\text { Estudo de caso E) } \\
\text { Simulação de } \\
\text { crescimento urbano }\end{array}$ & $\begin{array}{c}\text { Apoio a tomada de decisão de locais } \\
\text { estratégicos para equipamentos } \\
\text { públicos e simulação mais precisa de } \\
\text { tempo de retorno de equipamentos }\end{array}$ & $\begin{array}{c}\text { Coleta de dados precisos } \\
\text { da população com } \\
\text { intenções de origem e } \\
\text { destino }\end{array}$ \\
\hline $\begin{array}{c}\text { Estudo de caso Fl } \\
\text { Cálculo da energia solar } \\
\text { recebida }\end{array}$ & $\begin{array}{c}\text { Estimativa de necessidade de } \\
\text { condicionadores de ar auxiliando na } \\
\text { previsão de demanda }\end{array}$ & $\begin{array}{c}\text { Estimar o consumo da } \\
\text { população do local do } \\
\text { estudo }\end{array}$ \\
\hline
\end{tabular}

Fonte: Os autores (2019)

\section{CONCLUSÕES}

A partir do estudo realizado conclui-se que Cidades Inteligentes e CIM possuem conceitos e aplicações diversas que podem ser consideradas como complementares e estreitamente interligados. Dessa forma, a plataforma CIM demonstra-se potencialmente importante, pois 
possui uma base de dados modelados da infraestrutura urbana que, quando aliada às TICs, tem grandes potencialidades para a implementação de estratégias que tornem a gestão de cidades consolidadas mais inteligente.

Com possibilidades para ser implementadas em diferentes contextos com diversas ambições, como nos exemplos de aplicações estudados, afim de se ter informações em tempo real do que acontece na cidade e simular eventos futuros - podendo então prever o desfecho de determinada ação e embasar decisões -, estas abordagens estão sendo discutidas como uma estratégia para otimização da gestão urbana no século XXI.

Por fim, ressalta-se que, para o estabelecimento de uma abordagem combinada de CIM e Cidades Inteligentes, é necessário um corpo técnico especializado trabalhando de forma integrada e colaborativa, não apenas na construção e modelagem inicial da infraestrutura urbana, mas ao longo de todo o processo de gestão.

\section{AGRADECIMENTOS}

O presente trabalho foi realizado com apoio da Coordenação de Aperfeiçoamento de Pessoal de Nível Superior - Brasil (CAPES) - por meio do Programa de Apoio à Pós-Graduação (PROAP).

\section{REFERÊNCIAS}

ABELLA, A.; ORTIZ-DE-URBINA-CRIADO, M.; DE-PABLOS-HEREDERO, C. A model for the analysis of data-driven innovation and value generation in smart cities' ecosystems. Cities, v. 64, p. 4753, 2017.

ALMEIDA, F.; ANDRADE, M. L. CIM ou não? Considerações sobre City Information Modeling. In: Anais IV ENANPARQ. Porto Alegre, 2016. Disponível em:

$<$ https://www.researchgate.net/publication/305766451_CIM_ou_nao_Consideracoes_sobre_ City_Information_Modeling, 2016>. Acesso em: 15 de abril de 2019.

AMORIM, A. L. Estabelecendo requisitos para a Modelagem da Informação da Cidade (CIM). In: Anais IV ENANPARQ. Porto Alegre, 2016a. Disponível em:

<https://www.researchgate.net/publication/305701488_Estabelecendo_requisitos_para_a_M odelagem_da_Informacao_da_Cidade_CIM>. Acesso em: 15 de abril de 2019.

AMORIM, A. L. Cidades Inteligentes e City Information Modeling. In: Anais XX Congreso de la Sociedad Iberoamericana de Gráfica Digital (SIGraDi). Buenos Aires, 2016b. v. 3 n. 1, p. 481488.

AMORIM, A. L. Discutindo City Information Modeling (CIM) e conceitos correlatos. Gestão \& Tecnologia de Projetos, v. 10, n. 2, p. 87-100, 2015.

GIL, A. Como Elaborar projetos de pesquisa, 5a Edição, São Paulo: Editora Atlas, 2010.

KHEMLANI, L. City Information Modeling. AECbytes, 2016. Disponível em: <http://www.aecbytes.com/feature/2016/CitylnformationModeling.html>. Acesso em: 11 de abril de 2019.

KOMNINOS, N., BRATSAS, C., KAKDERI, C.; TSARCHOPOULOS, P. Smart City Ontologies: Improving the effectiveness of smart city applications. Journal of Smart Cities, vol.1, p. 1-16, 2015.

MIGUEL, P. A. C. Metodologia de Pesquisa em Engenharia de Produção e Gestão de

Operações. 2. ${ }^{a}$ ed. Rio de Janeiro: Elsevier, 2012. 
REITZ, T.; SCHUBIGER-BANZ, S. The Esri 3D city information model. In: Proceedings 8th International Symposium of the Digital Earth (ISDE8). Malásia, 2014.

SCHIEFELBEIN, J.; JAVADI, A.; LAUSTER, M.; REMMEN, P.; STREBLOW, R.; MULLER, D.

Development of a city information model to support data management and analysis of building energy systems within complex city districts. In: Proceedings International Conference CISBAT 2015 Future Buildings and Districts Sustainability from Nano to Urban Scale. Lausanne, 2015. p. 949-954.

SHERR, I. Chicago maps its underground maze. CNet, 2017. Disponível em:

<https://www.cnet.com/news/chicago-maps-its-underground-maze-rahm-emanuel/>. Acesso em: 19 de abril de 2019.

THOMPSON, E. M.; SMITH, K. M.; CHARLTON, J.; DOLNÍK, M. Planners in the future city: using city information modelling to support planners as market actors. Urban Planning, v. 1, n. 1, p. 7994,2016

UNION, I. T. Focus Group on Smart Sustainable Cities, 2015. Disponível em: <http://www.itu.int/en/ITU-T/focusgroups/ssc/Pages/default.aspx>. Acesso em: 05 de abril de 2019.

WEISS, M. C.; BERNARDES, R. C.; CONSONI, F. L. CIDADES INTELIGENTES: casos e perspectivas para as cidades brasileiras. Revista Tecnológica da Fatec Americana, v. 5, n. 1, p. 01-13, 2017.

XU, X.; DING, L.; LUO, H.; MA, L. From Building Information Modeling to City Information Modeling. Journal of Information Technology in Construction, v. 19, p. 292-307, 2014. 